\title{
Letter to the editor:
}

Re: Noel, et al. Oversensing issues leading to device extraction: when subcutaneous implantable cardioverter defibrillator reached a dead-end.

Christopher Monkhouse BSc CCDS, Pier Lambiase PhD FRCP FHRS

Barts Heart Centre, West Smithfield, London, EC1A 7BE

Corresponding author:

Professor Pier D. Lambiase, PhD, FRCP, FHRS

Barts Heart Centre, West Smithfield, London, EC1A 7BE

p.lambiase@ucl.ac.uk

$+44(0) 2034655855$

Word Count: 398

\section{Conflicts of interest \& Acknowledgments:}

PDL is supported by Educational grants and speaker fees from Boston Scientifiic, Medtronic and Abbott. Research is supported by UCL/UCLH Biomedicine NIHR and Barts BRC.

$\mathrm{CM}$ has received speaker fees from Abbott. 
We read with keen interest the article by Noel et.al regarding oversensing issues with the subcutaneous implantable cardioverter defibrillator (S-ICD) but have a number of concerns regarding the high rates of oversensing and the fact devices had to be extracted in $5.6 \%$ of cases rather than pre-operatively screen more intensively to ensure maximum sensing vector choices (right/sternal) or consider lead/generator repositioning early and even pre-emptively to overcome oversensing issues.

The majority of the cases had Brugada or ARVC when RBBB can present a challenge but 3 had myopotential oversensing treated by extraction. In our experience of 280 cases over 4 years we have only had to extract 1 device (0.4\%) due to oversensing issues (HCM patient) who had optimal left sternal lead placement and an intramuscular can but developed TWOS in primary and secondary vectors \& myopotential oversensing in alternate vector with inadequate sensing on the right side of the sternum.

We have published one case of oversensing causing an AF detection in a high BMI patient, which highlighted suboptimal lead placement prior to inappropriate therapy. Did the patients with oversensing have a high BMI or have detections by the AF monitor that could have provided an early warning of device sensing issues and pre-empt inappropriate shocks?

The SMART pass filter also can disable itself if specific criteria are met, had the device deactivated the SMART pass filter for the episodes recorded? We have both reprogrammed sensing vectors and repositioned leads/generators pre-emptively in some cases based on this early warning. Noel et.al discuss placing the device sub-muscularly - a critical alternative option for $3(50 \%)$ of the patients who had explantation for myopotentials.

We apply a strict screening and implant protocol to minimise oversensing, including screening both sides of the sternum as well as exercise testing in channelopathy and cardiomyopathy cases to maximise the number of vector options pre-implant. Were any of the patients assessed for right sided lead placement and was exercise testing used pre-operatively to look for QRS and T wave 
morphology changes? This would have been especially important in the 2 cases who had a VT ablation which could have had deleterious effects on the sensing vectors and lead repositioning could have been considered.

We strongly recommend close monitoring to pre-empt these problems and intense pre-implant screening to maximise options available in dealing with these complex cases to avoid the dead-end of an S-ICD explant. 